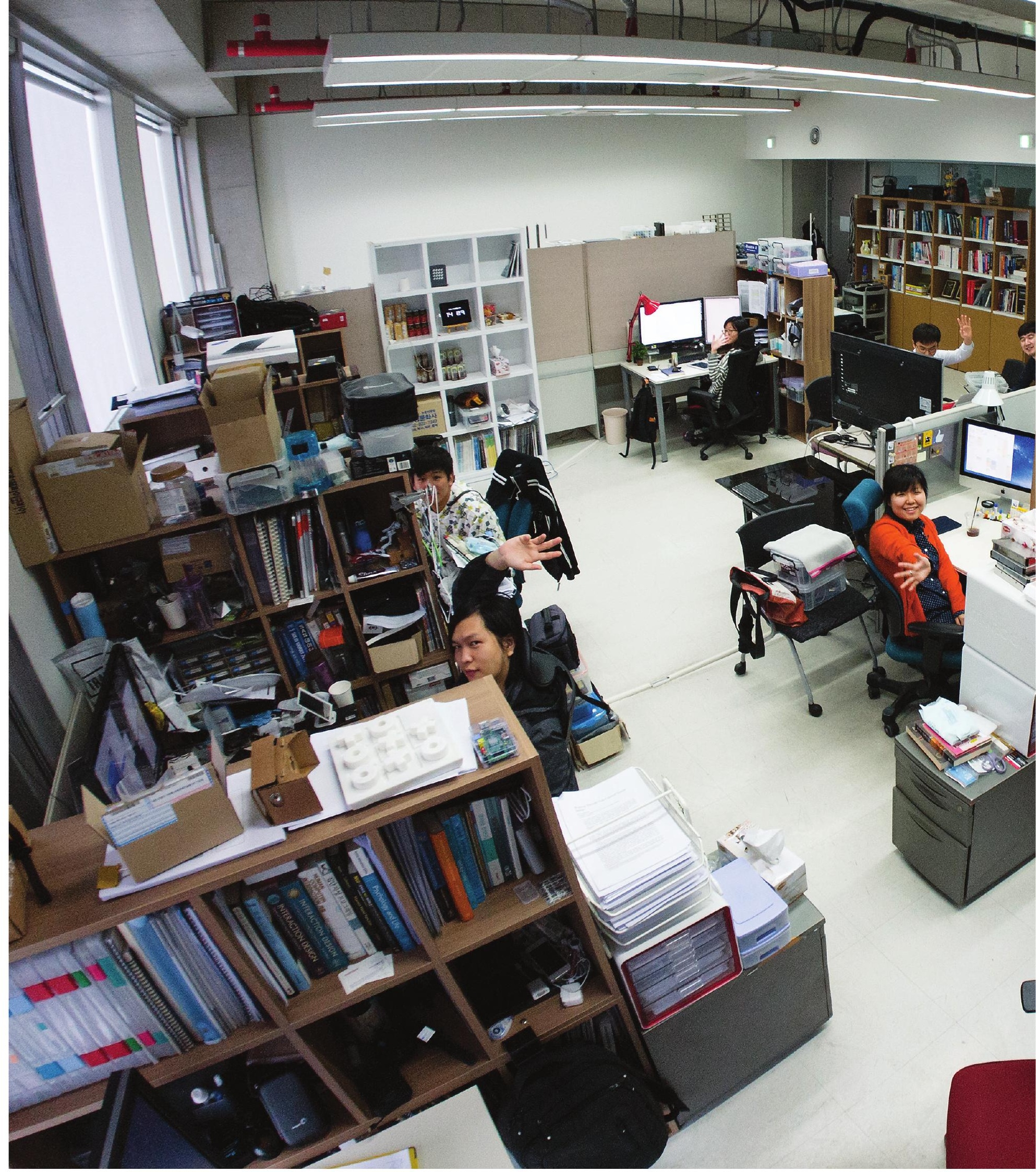




\section{Human-Computer Interaction Lab @KAIST}

As told by Seongkook Heo, Sunjun Kim, Jaehyun Han, Jiseong Gu, Jaeyeon Lee, Jeongmin Son, Jin Gun Jung, Eunhye Youn, Sunggeun Ahn, and Geehyuk Lee

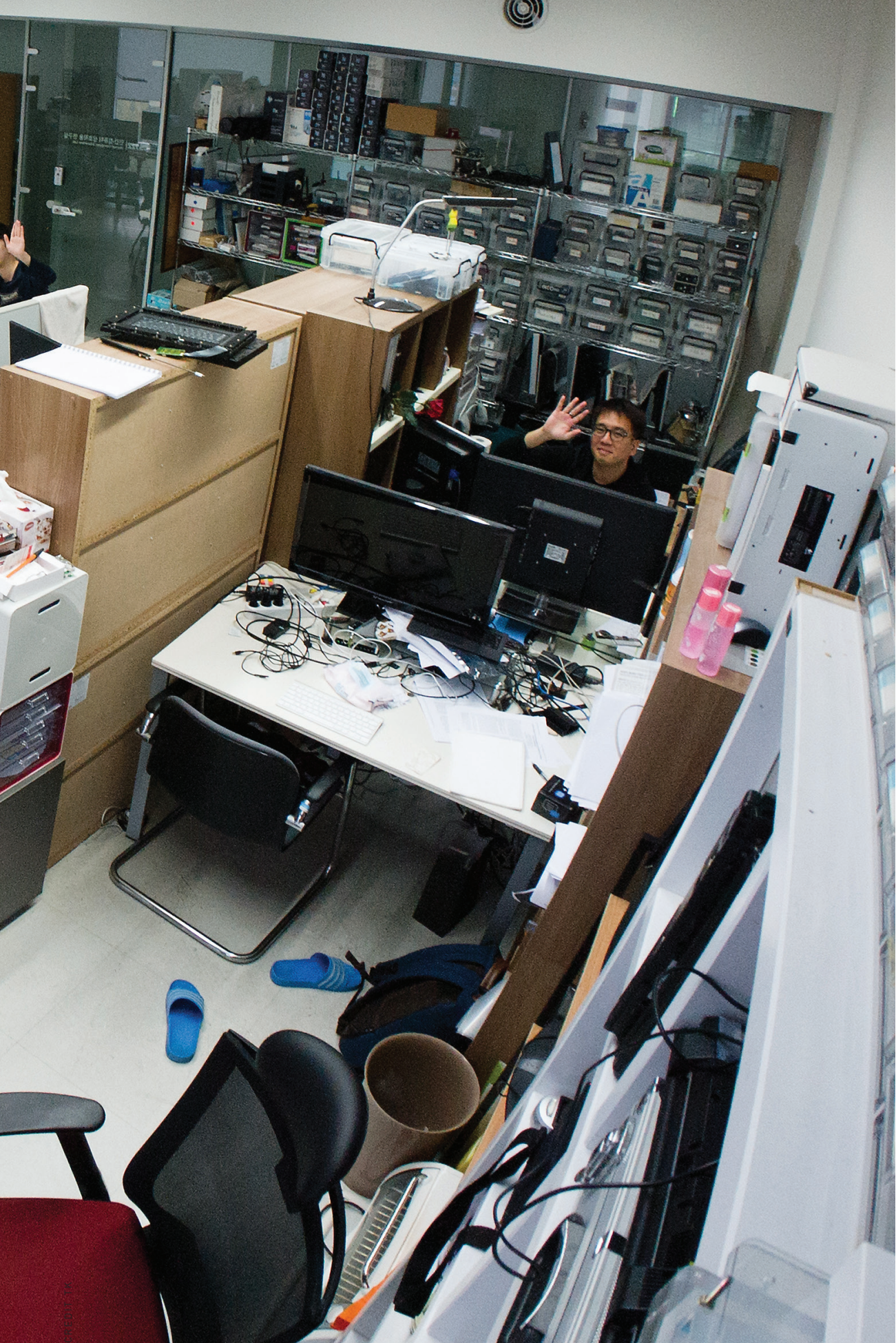

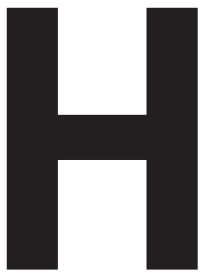
ow do you describe your lab to visitors? The name HCI Lab is in fact too big for our small lab. Among various areas in HCI, we mainly work on new

interaction techniques for new types of computers. While we are a computer science lab, we also make new hardware and hack existing hardware to explore design possibilities that go beyond the constraints of current hardware. Our present focus is on the user interface problems introduced by new computing gadgets such as smartphones and smart TVs.

How many people are in your lab, and what is the mix of backgrounds and roles?

We have one professor, seven doctoral students, and two master's students. Our undergraduate degrees are diverse: four majored in CS, four in electrical engineering, one in physics, and one in brain engineering. However, all of the students are currently in a graduate program in CS, so we all have a background in core computer science. The members have preferences for different roles, but everyone does everything, from prototyping to evaluation.

Describe a day in the life of your lab. A lab rule is that everybody should come to the lab by $10 \mathrm{a} . \mathrm{m}$. There is no rule about leaving. What we do during our work hours has a weekly cycle. On Mondays and Tuesdays, each student has an individual meeting with our advisor. On Wednesdays, we have a two-session lab seminar: one session for reviewing papers and the other for anything interesting. On Thursdays, we have group meetings for funded research projects, and on Fridays, we clean our lab together.

\section{Describe how people interact in your lab.}

There are regular meetings where all lab members gather to talk about our research. There are also ad hoc meetings, such as brainstorming sessions that students can call when they need feedback from other colleagues. The most interesting and fruitful conversation, however, occurs when we eat together during lunch, dinner, and coffee hour. Korea is 


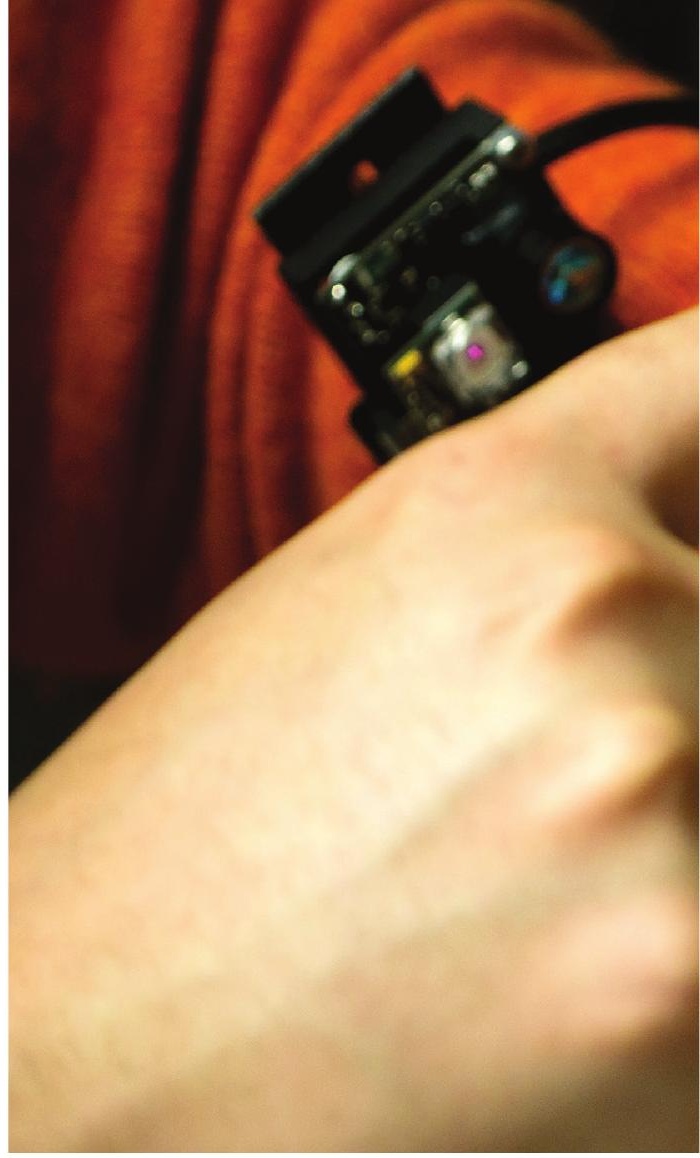

$\rightarrow$ Transture: a touch gesture on a small screen continued into the air.

$\rightarrow$ Trampoline: a double-sided, elastic-surface input device for relievo modeling.

known to have a relatively high powerdistance index, but we tend to forget this around a meal.

\section{What is a unique feature of your lab?}

We like making things, software or hardware. We sometimes make things just because we feel like making them - without a clear purpose. Some of these things eventually lead to a paper, but more often they fail to find a good use and become a mere display in the lab. This is why we like venues such as the UIST Student Competition and the SIGGRAPH Emerging Tech exhibition. This is in fact a tradition of our lab that we cherish. During interviews with a new student, the first thing that our adviser asks is, "Do you like to make things?”

\section{What is one feature of your lab you could not do without?}

A feature that makes us stand out from other CS labs is a workshop for prototyping. The workshop is small but has everything needed for making and trimming prototypes: a $3 \mathrm{D}$ printer, a laser cutter, a CNC machine, an electronics workbench, and many other small tools. The workshop has accumulated many leftovers from numerous past prototyping activities, including various electronic parts and mechanical debris in various shapes, and has been optimized for our specific prototyping needs.

\section{What is one feature of your lab that you want and do not have?}

As most any university lab would answer, we would love to have more space for our prototyping activities and experiments. A more specific wish, as a lab in Korea, would be the ability to freely express our thoughts in English. Most of us have difficulty thinking in English, so we often write the outline of our papers in Korean and then translate it into English.
And, in most cases, the result is not satisfactory. So, fluency in English is the skill that we miss most, especially when approaching a CHI deadline.

\section{What is the one thing you see as most important about what you do there?}

Attempting many things and experiencing many failures. One student tried six different ideas before an idea finally resulted in a paper. Too often we feel that we should be more efficient; however, we also believe that a university laboratory should be an environment that can embrace failure for creative challenges. Investing time to elaborate on crude ideas and sharing the experience of failure are the most privileged things we do. (This is what our advisor tells us, and we all want to graduate early.)

http://hcil.kaist.ac.kr 

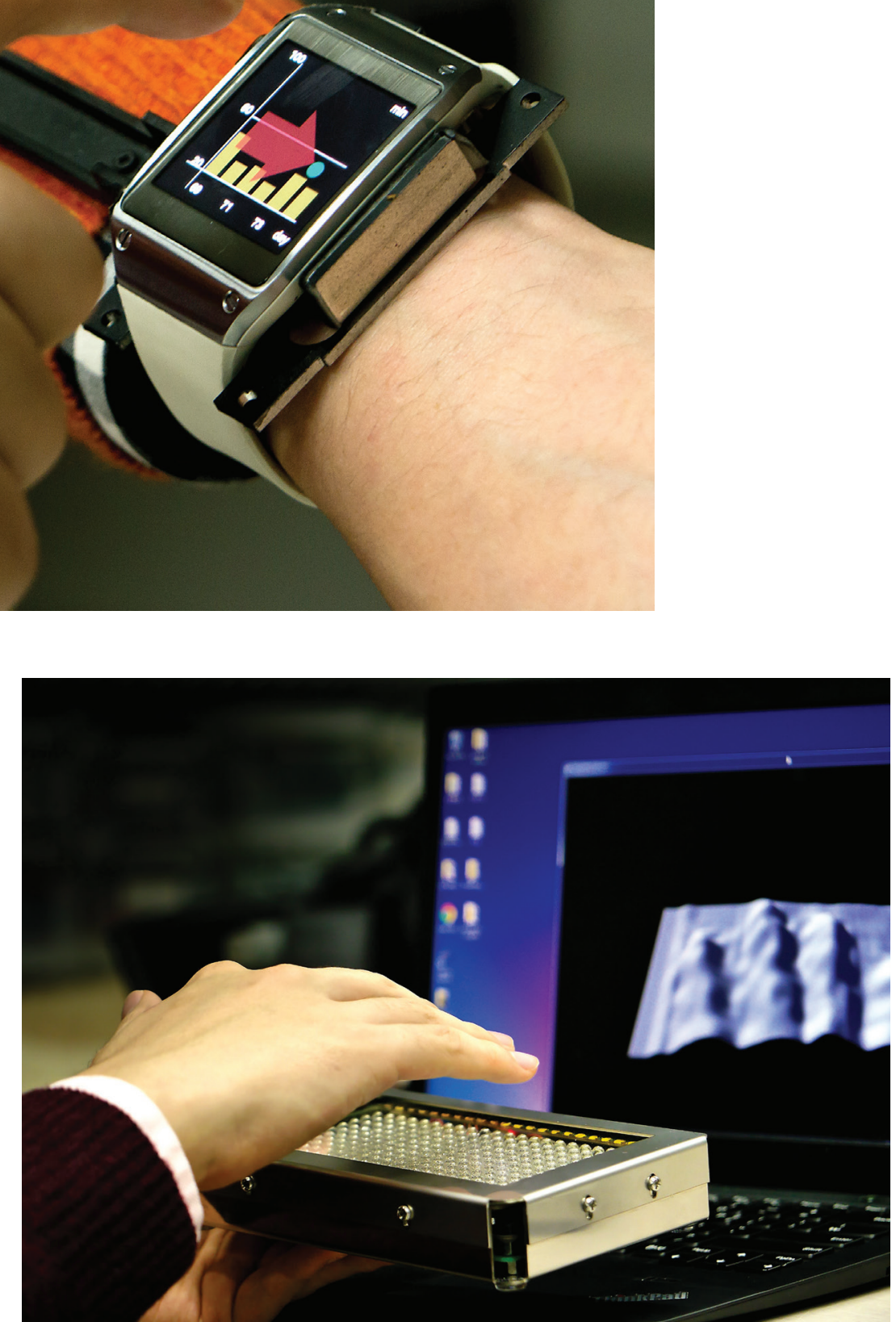

$\rightarrow$ ScreenPad: an optical touchpad that senses fingers on and over the surface.
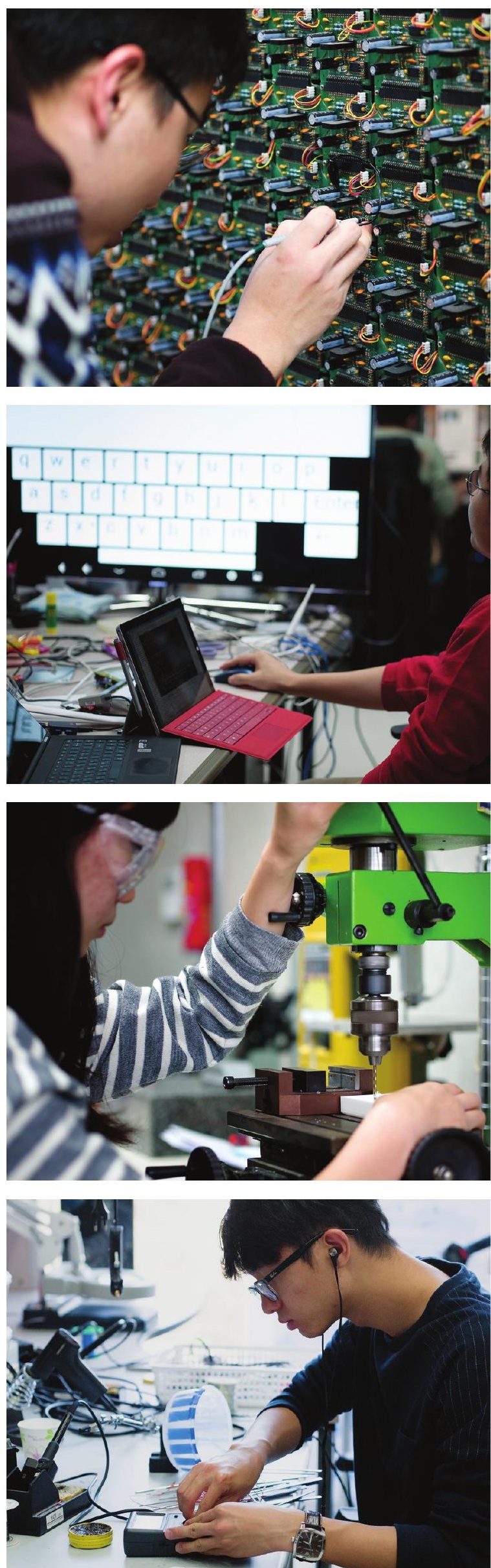

Korea is known to have a relatively high powerdistance index, but we tend to forget this around a meal. 